\title{
Quantitative analysis of cell-free DNA by droplet digital PCR reveals the presence of EGFR mutations in non-malignant lung pathologies
}

\author{
Rajesh Venkataram ${ }^{1}$, Srividya Arjuna ${ }^{2}$, Giridhar Belur Hosmane ${ }^{1}$, Anirban Chakraborty ${ }^{2}$ \\ ${ }^{1}$ Department of Pulmonary Medicine, KS Hegde Medical Academy, Nitte (Deemed to be University), Mangalore; \\ ${ }^{2}$ Division of Molecular Genetics and Cancer, Nitte University Centre for Science Education and Research, Nitte (Deemed \\ to be University), Mangalore, India
}

\begin{abstract}
Correspondence: Dr. Anirban Chakraborty, PhD, Professor, Division of Molecular Genetics and Cancer, Nitte University Centre for Science Education and Research, (NUCSER), Nitte (Deemed to be University), Mangalore 575018, India.

Tel. +91.8242204292. E-mail: anir.abc@gamil.com

Acknowledgements: We would like to thank Nitte (Deemed to be university), Mangalore, India for the financial support in the form of a research grant (NUFR11/2017/06/08). We would like to acknowledge the help rendered by Dr. Kishan Prasad H.L., Department of pathology, KS Hegde Medical Academy, in obtaining histopathological and cytological images. We also thank Dr T. S. Ganesan, Cancer Institute (WIA), for his critical inputs and suggestions on the manuscript.

Key words: Liquid biopsy; EGFR mutations; ddPCR; NSCLC; nonmalignant lung pathologies.

Contributions: RV, conceptualization, data curation, formal analysis, funding acquisition, investigation, methodology, project administration, software, validation, visualization, original draft; SA, formal analysis, investigation, methodology, visualization; GB, data curation, project administration; AC, conceptualization, formal analysis, project administration, resources, software, supervision, validation, visualization, review and editing. All the authors have read and approved the final version of the manuscript and agreed to be accountable for all aspects of the work.
\end{abstract}

Conflicts of interest: The authors declare that they have no conflicts of interest in regard to this study.

Funding: The study was funded by an Intramural Research Grant (NUFR11/2017/06/08) awarded to RV and AC by Nitte (Deemed to be University).

Ethical Approval and consent to participate: The study was approved by the Central Ethics Committee of Nitte (Deemed to be University Approval ID: NU/CEC/2017-18/0127).

Informed consent was obtained from all individual participants included in the study.

Availability of data and material: The clinical data and the study materials are available 2 from the corresponding author on reasonable request.

Received for publication: 24 December 2020.

Accepted for publication: 18 April 2021.

${ }^{\circ}$ Copyright: the Author(s), 2021

Licensee PAGEPress, Italy

Monaldi Archives for Chest Disease 2021; 91:1748

doi: 10.4081 /monaldi.2021.1748

This article is distributed under the terms of the Creative Commons Attribution Noncommercial License (by-nc 4.0) which permits any noncommercial use, distribution, and reproduction in any medium, provided the original author(s) and source are credited.

\begin{abstract}
Tyrosine kinase inhibitors (TKIs) targeting epidermal growth factor receptor (EGFR) are effectively used in treatment of nonsmall cell lung cancer (NSCLC). Mutation profile of tyrosine kinase domain of EGFR determines the eligibility of the patients for tyrosine kinase inhibitor (TKI) therapy. Liquid biopsy, which relies on circulating tumor-derived nucleic acids, has emerged as an effective tool in lung cancer management with proven diagnostic, prognostic and predictive applications. We screened 100 subjects, suspected to have lung malignancy, for four hotspot mutations including three activating (G719S, Ex19del E746-A750 and L858R) and one acquired (T790M, de novo) in EGFR gene by droplet digital PCR (ddPCR). While 97 subjects were subsequently confirmed to have lung malignancy based on histo/cytopathological studies, three cases turned out to be non-malignant lung pathologies that were completely cured by antibiotic therapy. Intriguingly, ddPCR revealed the presence of EGFR mutations in these non-malignant subjects. Two cases showed the presence of G719S and T790M mutations respectively and another had compound mutations (T790M and L858R). The detection of EGFR mutations in nonmalignant pulmonary conditions opens up a new area of research.
\end{abstract}

\section{Introduction}

Lung cancer is one of the major malignancies worldwide and non-small cell lung cancer (NSCLC) is the major subtype, accounting for approximately $85 \%$ of the cases $[1,2]$. NSCLC harbouring specific mutations in epithelial growth factor receptor (EGFR) gene and anaplastic lymphoma kinase (ALK) gene have been shown to be highly sensitive to targeted therapy in the form of TKI (Tyrosine Kinase Inhibitors) [3-5]. Within EGFR, exons 18-24 code for the tyrosine kinase domain. However, mutations that interfere with the response to TKIs are restricted to exons 18-21 [6]. Among the frequently observed EGFR mutations that confer sensitivity to TKIs, small in-frame deletions in exon 19 and point mutations in exon 21 causing a leucine to arginine substitution at codon 858 (L858R) are the "hotspots", comprising about $85-90 \%$ of all such EGFR mutations [7]. The remaining $10 \%$ come under the category of uncommon sensitizing mutations, of which G719X, point mutation at codon 719 that results in substitution of glycine with alanine (G719A), cysteine (G719C) and serine (G719S), accounts for approximately $5 \%$ of the mutations [8]. On the other hand, mutations associated with TKI resistance are concentrated in exon 20, with point mutation at codon 790 resulting in substitution of tyrosine with methionine (T790M) accounting for nearly $50 \%$ of all such mutations [7].

The term "liquid biopsy" refers to detection of cancer from 
blood or any other body fluid. It relies on the detection and characterization of tumor derived substances, such as circulating tumor DNA (ctDNA), and circulating tumor cells (CTCs), which are present in the plasma or serum $[9,10$.] Identification of ctDNA has been a challenge for many decades because; cancer-associated mutations often evade detection due to their low concentrations relative to the background of wild type DNA in a given sample. However, the recent discovery of the droplet digital PCR (ddPCR) has overcome this difficulty and this technique is now being used for detection of EGFR mutations in ctDNA obtained from lung cancer subjects [11-14]. Droplet digital PCR works on the principle of "partitioning effect" where DNA is compartmentalized into water-oil emulsion droplets through microfluidics, which are then amplified individually to provide absolute quantification of the data and expression of values as copies per microlitre of the sample. This ultra-sensitive tool provides real-time, non-invasive monitoring of tumor- derived mutant alleles of interest present at extremely low concentrations in plasma and allows for prediction of response to targeted therapy [5].

While there is abundant literature on detection of EGFR mutations in NSCLC subjects by ddPCR, reports on the presence of such mutations in subjects with non-malignant lung pathologies is extremely limited. Studies of such mutations in subjects having adenocarcinoma of lung with past history of pulmonary tuberculosis have been reported $[15,16]$. Here we report the presence of EGFR mutations in non-malignant lung pathologies including a case of an active tuberculosis infection.

\section{Methods}

\section{Sample collection}

One hundred consecutive subjects suspected to have lung malignancy based on characteristics like clinical features; radiological features and lesion morphology during bronchoscopy were included in the study. Five $\mathrm{ml}$ of venous blood was collected in EDTA coated vacutainers after obtaining informed consent. Plasma was isolated within 2 hours of collection by centrifuging the blood samples at $3500 \mathrm{rpm}$ for $10 \mathrm{~min}$. Subjects who were already under treatment for lung cancer and those who had prior history of malignancy were not included. Final diagnosis was based on histopathological / cytopathological studies. The study protocol was approved by the Central Ethics Committee of the University.

\section{cfDNA extraction}

cfDNA was extracted from plasma using Qia-Amp circulating nucleic acid kit, (Qiagen, Germany) in accordance with the manufacturer's instructions.

\section{Droplet digital PCR assay}

ddPCR analysis was performed using QX 200 Droplet Digital PCR System (Bio-Rad, USA) as per the protocol for PrimePCR ddPCR Mutation Detection Assays, validated for both FAM and HEX-labelled probes. The assays are such designed that the FAM probe binds to the mutant allele whereas the HEX probe binds to the wildtype allele. Four mutations namely EGFR Exon 18 G719S, EGFR Exon 19 del, EGFR Exon 20 T790M, and EGFR Exon 21 L858R, were screened using commercially available assays from Bio-Rad. The ddPCR protocol includes four steps. Step 1 is preparation of master mix containing the ready-to-use primer-probe mix with known amount of cfDNA (45-60 ng/sample). Step 2 includes loading the individual samples onto 8-well microfluidic cartridge and mixing with oil to generate the droplets. Step 3 is amplification of individual droplets in a thermocycler and step 4 is reading the fluorescence of individual droplets in two channels (FAM and HEX) by the droplet reader at the end of the amplification. The results are expressed as number of mutant copies / $\mu$ l of blood.

\section{Results}

As expected, ddPCR revealed the presence of four hotspot mutations in varying combinations in the 97 confirmed cases of lung cancer. However, ddPCR also revealed that the 3 cases, which were conclusively proven to be non-malignant pathologies after double confirmation by two different pathologists, harboured hot spot mutations in EGFR gene. The clinical details of these 3 subjects are shown in Table 1. Interestingly, all the three cases had history of cigarette smoking.

The first subject, coded as non-malignant lung pathology-1 (NMLP-1), was positive for Mycobacterium tuberculosis and showed the presence of G719S mutation with a concentration of 20.6 copies/ $\mu 1$ of blood. As shown in Figure 1, the 1D amplitude of G719S assay for NMLP- 1 (column D04), revealed the presence of blue droplets (FAM positive, upper panel), which indicates the presence of mutant copies in the sample. The column G04 is the positive control (plasmid DNA with G719S mutation), which expectedly showed a large number of blue droplets. The lower panel shows the 1D amplitude of HEX positive droplets (green droplets), indicating the presence of wild type copies in both patient and positive control (D04 and G04). The column H04 is the no template control, which did not show the presence of blue or green droplets.

The second subject, coded as NMLP-2, was confirmed as having lung abscess and showed the presence of T790M mutation (de novo) with a concentration of 15.4 copies/ $\mu$ l of blood. Figure 2 shows the 1D amplitude of T790M assay for NMLP-2 (column F01) compared to the no template control (column H05). As evident from the image, the presence of blue droplets in the F01 column indicates FAM positive droplets (mutant copies) in the sample.

The third subject, coded as NMLP-3, was a case of inflammatory lesion and harboured L858R mutation with a concentration of 3.7 copies/ $\mu 1$ of blood (Figure 3A). As seen in the image, column C06, which contained the patient sample showed the presence of FAMpositive (blue droplets), indicating the presence of L858R mutant copies compared to the no template control (column A03). Interestingly, NMLP-3 also showed the presence of T790M de novo mutation at a concentration of 1.3 copies/ $\mu$ l of blood. As shown in Figure 3B, column D06, which contained the patient sample showed the presence of FAM-positive droplets (blue droplets), which are positive for T790M mutation. The column E06 contained the positive control DNA (plasmid DNA with T790M mutation).

The spectrum of the mutation status for each subject is shown in Table 2. The ratio of FAM positive droplets (mutant allele) to HEX droplets (wild type allele) and the fractional abundance of the mutant copies for these 3 subjects are shown in Table 3.

\section{Discussion}

Mutations in EGFR tyrosine kinase domain (exon 18 to 24) play a vital role in predicting the response to TKIs in NSCLC. Among the many mutations, identified, some are considered as 
Table 1. Clinical, radiological and diagnostic characteristics and treatment outcome.

\begin{tabular}{|c|c|c|c|c|c|c|c|}
\hline $\begin{array}{l}\text { Subject } \\
\text { code }\end{array}$ & Symptoms & $\begin{array}{l}\text { Smoking } \\
\text { status }\end{array}$ & Age & $\begin{array}{l}\text { Radiological } \\
\text { features }\end{array}$ & $\begin{array}{l}\text { Mode of } \\
\text { diagnosis }\end{array}$ & $\begin{array}{l}\text { Histopatholgical/ } \\
\text { cytological features }\end{array}$ & $\begin{array}{l}\text { Final } \\
\text { outcome }\end{array}$ \\
\hline NMLP-1 & $\begin{array}{l}\text { Chronic cough, } \\
\text { weight loss }\end{array}$ & Smoker & 52 & $\begin{array}{l}\text { Mass in right lower } \\
\text { lobe with effusion }\end{array}$ & $\begin{array}{l}\text { Endobronchial } \\
\text { biopsy from the } \\
\text { growth in right } \\
\text { ower lobe bronchus, } \\
\text { BAL: AFB \& CBNAAT }\end{array}$ & $\begin{array}{l}\text { Biopsy: granuloma } \\
\text { with Langhans giant } \\
\text { cellsBAL, AFB \& } \\
\text { CBNAAT: } \\
\text { Positive for } \\
\text { Mycobacterium } \\
\text { Tuberculosis }\end{array}$ & $\begin{array}{l}\text { Cured with } 6 \text { months } \\
\text { of anti-tubercular } \\
\text { therapy }\end{array}$ \\
\hline NMLP-2 & $\begin{array}{l}\text { Chronic cough, } \\
\text { racture of the } \\
\text { hip suspected } \\
\text { to be a } \\
\text { Pathological } \\
\text { fracture }\end{array}$ & Smoker & 62 & $\begin{array}{l}\text { Mass in right upper } \\
\text { lobe }\end{array}$ & CT guided FNAC & $\begin{array}{l}\text { Cytology: } \\
\text { Sheets of neutrophils, } \\
\text { fibrous tissue, } \\
\text { bacterial colonies } \\
\text { suggestive of Lung } \\
\text { abscess }\end{array}$ & $\begin{array}{l}\text { Cured with } 3 \text { weeks } \\
\text { of antibiotic therapy }\end{array}$ \\
\hline NMLP-3 & Chronic cough & Smoker & 54 & $\begin{array}{l}\text { Mass in right lower } \\
\text { lobe }\end{array}$ & CT guided FNAC & $\begin{array}{l}\text { Cytology: } \\
\text { Inflammatory smear with } \\
\text { few atypical cells with high } \\
\mathrm{N}: \mathrm{C} \text { ratio Hepatoma to be } \\
\text { ruled outAFP \& liver imaging: } \\
\text { within normal limits }\end{array}$ & $\begin{array}{l}\text { Cured with } 3 \text { weeks } \\
\text { of antibiotic therapy }\end{array}$ \\
\hline
\end{tabular}

BAL, broncho-alveolar lavage; AFB, acid fast bacilli; CBNAAT, cartridge based nucleic acid amplification test; CT, computed tomography; FNAC, fine needle aspiration cytology; N:C ratio, nucleus: cytoplasm ratio; AFP, alfa feto protein.

Table 2. Case-wise EGFR hotspot mutation profile by ddPCR.

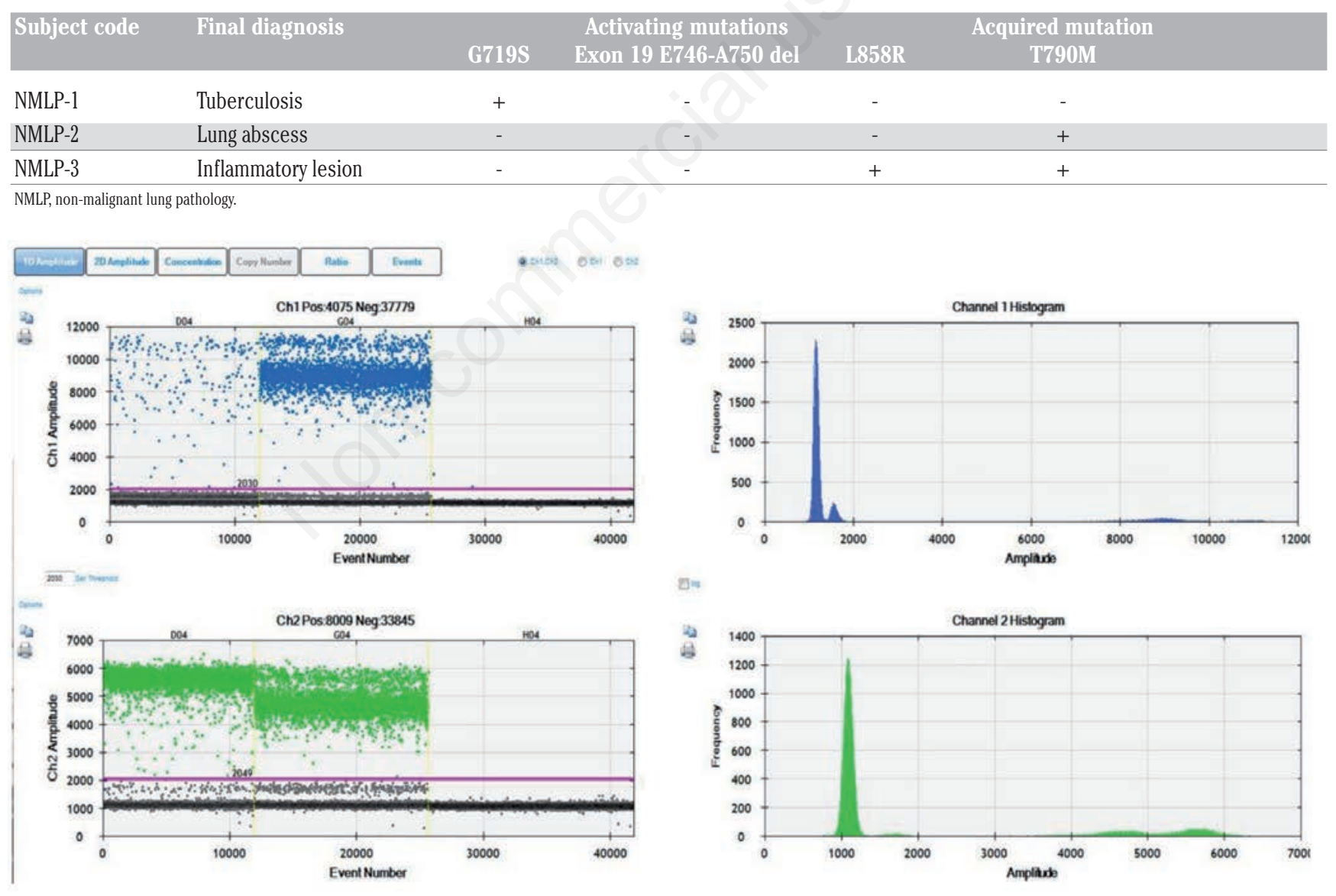

Figure 1. G719S mutation assay by ddPCR for NMLP-1. Snapshot of 1D amplitude obtained from the QuantaLife software in QX200 ddPCR platform. The assay included a set of primers and two competitive probes, one labelled with FAM (for G719S mutant allele, Channel 1) and another with HEX (for G719S wild type allele, Channel 2). The blue droplets (FAM positive) indicate the presence of mutant copies in the sample (column D04). Green droplets (HEX positive) indicate the wild-type copies. The black droplets below the threshold line (magenta) are the negative droplets having no DNA. The column G04 is the positive control (plasmid DNA) and the column $\mathrm{HO4}$ is no-template control. 
hotspots by virtue of their presence in a large number of subjects. Among the frequently encountered mutations, three activating mutations namely, EGFR Exon 18 G719S, EGFR Exon19 E746A750 del, EGFR Exon 21 L858R, which are associated with sensitivity to TKIs; one acquired mutation namely Exon 20 T790M associated with resistance to TKIs are routinely included for molecular testing of NSCLC.

In course of a molecular screening of a large cohort of lung cancer subjects, we identified 3 subjects in this study, who were initially suspected to have lung malignancy but were subsequently proven to have non-malignant pathologies. They were followed up till the primary pathology was cured with appropriate treatment as evidenced from imaging data (both pre- and post-treatment) showing spectacular response to these therapies. Our first case, NMLP1 , was a case of pulmonary tuberculosis diagnosed based on conventional (histopatholgical) and molecular methods (Figure 4D and Table 1). The patient recovered completely after anti- tubercular therapy (Figure 4 A-C) and at the end of 6 months, sputum smear for AFB (Acid Fast Bacilli) was also negative. However, the ddPCR results revealed a high load of G719S mutant copies of EGFR gene in the patient, a mutation which is generally known to be associated with malignancy of lungs.
Similarly, the second case (NMLP-2), which was finally diagnosed as lung abscess based on the cytological study of the aspirate from the lesion, which showed the presence of sheets of neutrophils (Figure 5B). The patient was put on empirical antimicrobial therapy and was completely asymptomatic following three weeks of treatment. The pre-treatment CT image is shown in Figure $5 \mathrm{~A}$. The causative bacteria could not be isolated as the specimen was not sent for microbiological studies because the appearance of aspirated material was not typical of pus and suspicion of malignancy was very high given the history of chronic cough, suspected pathological fracture in a chronic smoker. Intriguingly, this subject scored positive for T790M mutation, which is usually associated with acquired resistance to TKI manifesting after the commencement of the treatment with TKI. Germline transmission of T790M mutation in lung cancer subjects have been reported in patients with lung adenocarcinoma and it was suggested as a susceptibility trait for secondary somatic activating mutations [1719]. However, the presence of T790M in a non-malignant lung pathology is highly unusual and such a finding deserves further investigation on the possible molecular mechanisms involved.

The third case (NMLP-3), which was an inflammatory lesion (Figure 6C), similar to the second case, also scored positive for

Table 3. Ratio (FAM/HEX) and fractional abundance (FAM/FAM+HEX) of mutant and wild type droplets in plasma by ddPCR.

\begin{tabular}{llccc} 
Sulbject code & Final diagnosis & Target mutation & $\begin{array}{c}\text { Ratio } \\
\text { (FAM/HEX) }\end{array}$ & $\begin{array}{c}\text { Fractional abundance } \\
\text { (FAM/FAM+HEX) }\end{array}$ \\
NMLP-1 & Tuberculosis & G719S & 0.043 & 4.1 \\
NMLP-2 & Lung abscess & T790M & 0.23 & 18.7 \\
\hline NMLP-3 & Inflammatory lesion & L858R & 0.30 & 23.0 \\
NMLP-3 & Inflammatory lesion & T790M & 0.14 & 12.0 \\
\hline
\end{tabular}

NMLP, non-malignant lung pathology.
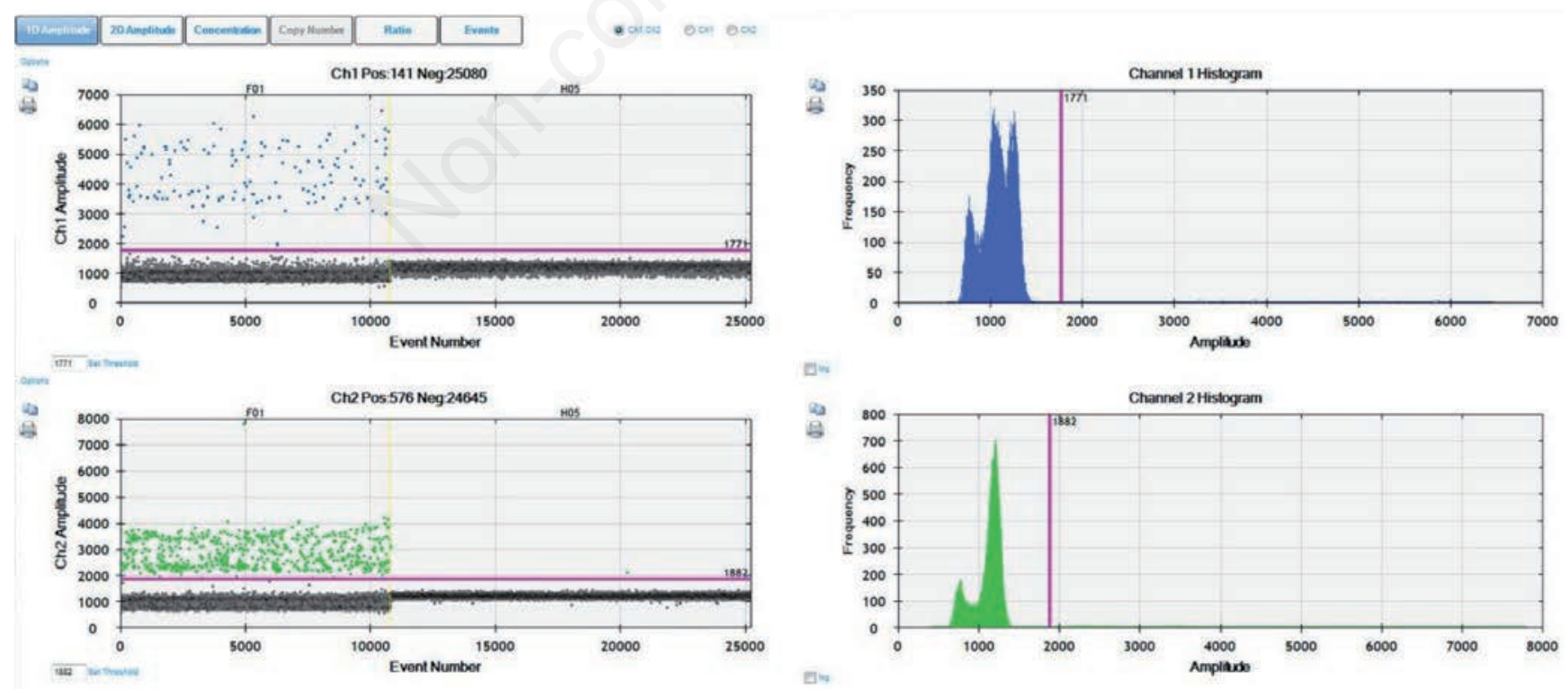

Figure 2. T790M mutation assay by ddPCR for NMLP-2. Snapshot of 1D amplitude obtained from the QuantaLife software in QX200 ddPCR platform. The assay included a set of primers and two competitive probes, one labelled with FAM (for T790M mutant allele, Channel 1) and another with HEX (for T790M wild type allele, Channel 2). The blue droplets (FAM positive) indicate the presence of mutant copies in the sample (column F01). Green droplets (HEX positive) indicate the wild-type copies. The black droplets below the threshold line (magenta) are the negative droplets having no DNA. The column H05 is no-template control. 

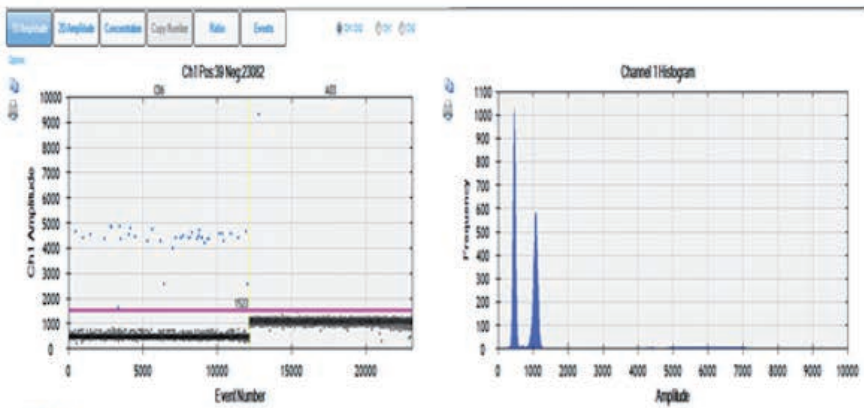

sine
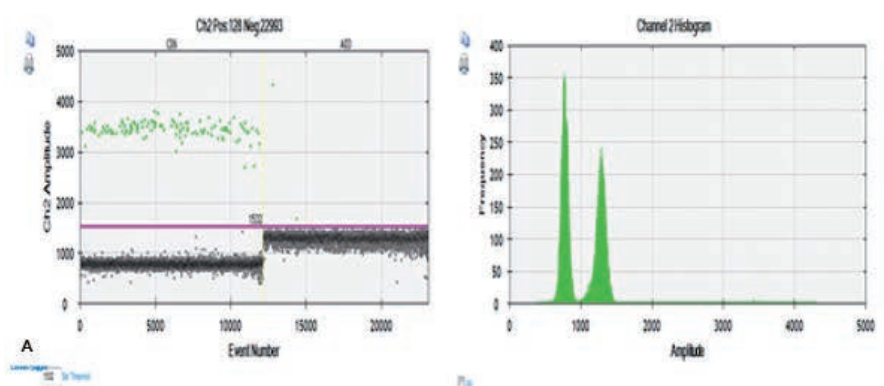
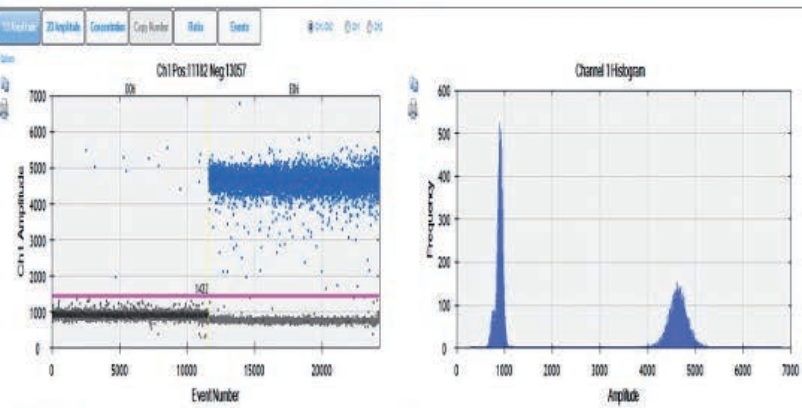

$\overline{\mathbf{c}} \mathrm{ne}$
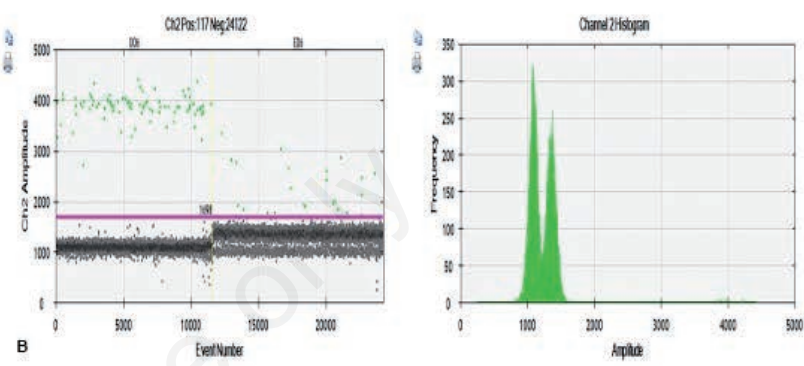

Figure 3. L858R and T790M mutation assays by ddPCR for NMLP-3. Snapshots of 1 D amplitudes obtained from the QuantaLife software in QX200 ddPCR platform for L858R (A) and T790M (B). The assays included a set of primers and two competitive probes, one labelled with FAM (for mutant allele, Channel 1) and another with HEX (for wild type allele, Channel 2). The blue droplets (FAM positive) indicate the presence of mutant copies in the sample (column C06 for L858R and D06 for T790M respectively). Green droplets (HEX positive) indicate the wild-type copies. The black droplets below the threshold line (magenta) are the negative droplets having no DNA. The column A03 in (A) is no-template control and E06 in (B) is positive control (plasmid DNA).
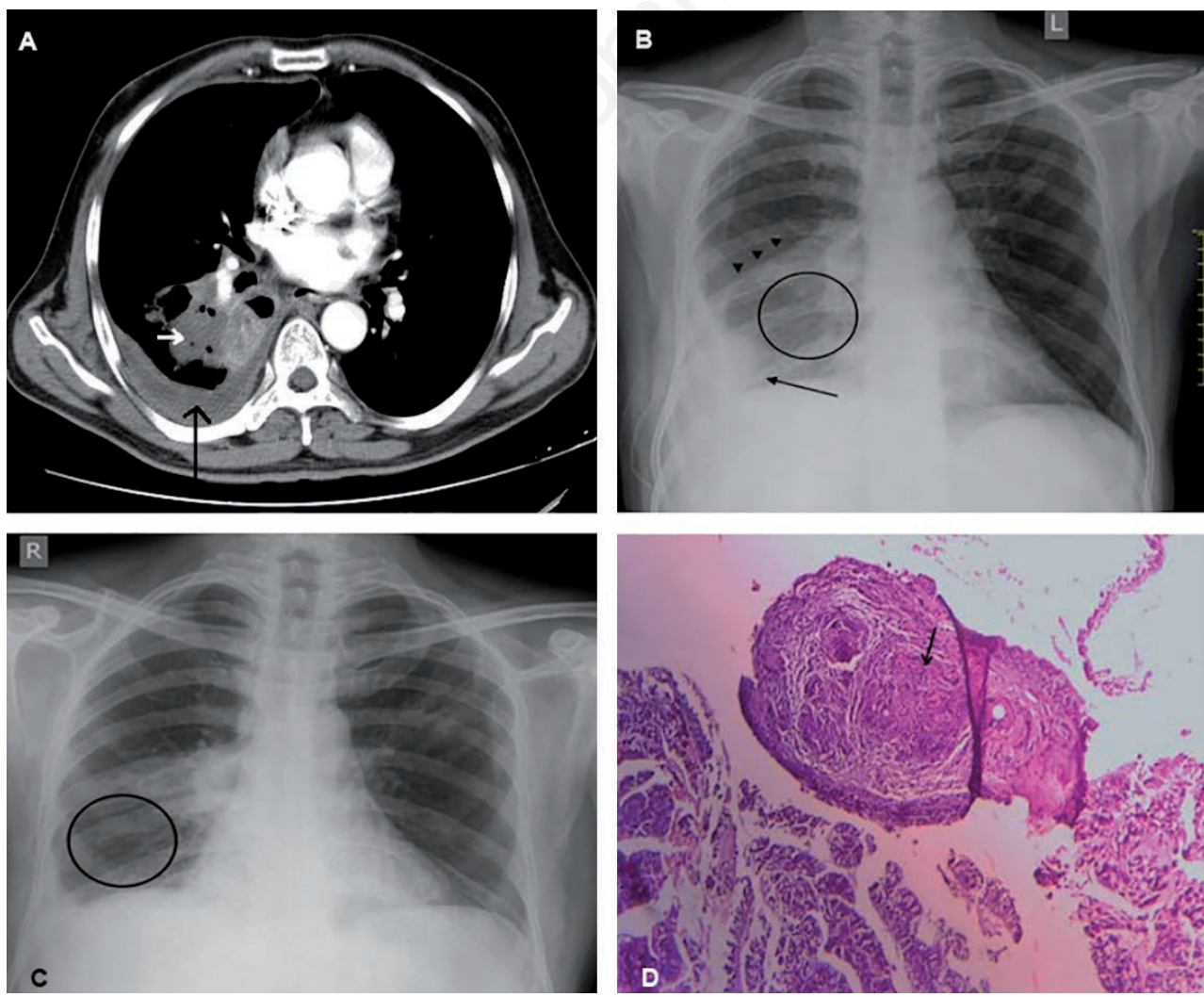

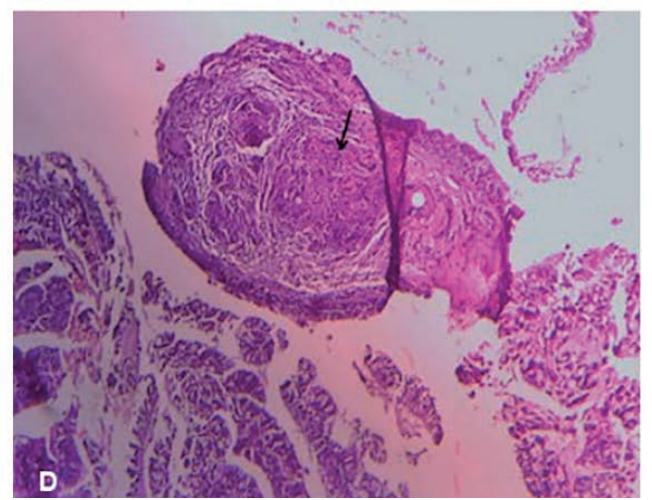

Figure 4. Imaging and histopathological features of NMLP-1. A) Axial CT image showing mass lesion in right lower lobe of the lung with collapse of the adjacent part of the lobe (white arrow). Pleural effusion is also present (black arrow). B) Chest $\mathrm{X}$ ray showing haziness of right lower lobe (black circle) indicating partial collapse (airlessness) resulting in pulling down of the oblique fissure (arrow heads); good amount of pleural effusion is also seen (black arrow). C) Chest $\mathrm{X}$ ray after 6 months of Antitubercular therapy showing well aerated lung (black circle) indicating significant reduction in the size of the mass lesion. Pleural effusion has significantly resolved. D) Histopathological image of the endobronchial biopsy of the mass lesion showing granuloma with Langhans giant cells (black arrow) suggestive of tubercular pathology. 
T790M mutation in addition to the presence of L858R mutation. As evident from the CT and X ray images (Figure 6 A-C), the patient showed near complete recovery after three weeks of antibiotic treatment. Though there was an element of suspicion of hepatoma being the lesion in question, all relevant investigations like Alfa Feto Protein (AFP) assay and imaging studies were not in favour of hepatoma. Presence of dual/multiple EGFR mutations in lung adenocarcinoma has been reported earlier [20], but evidence of dual EGFR mutations in an infectious condition of lung has not been reported so far. Previous studies have reported the presence of exon 19 deletion mutations in subjects with adenocarcinoma of lung that either had tuberculosis in the past or had radiological findings
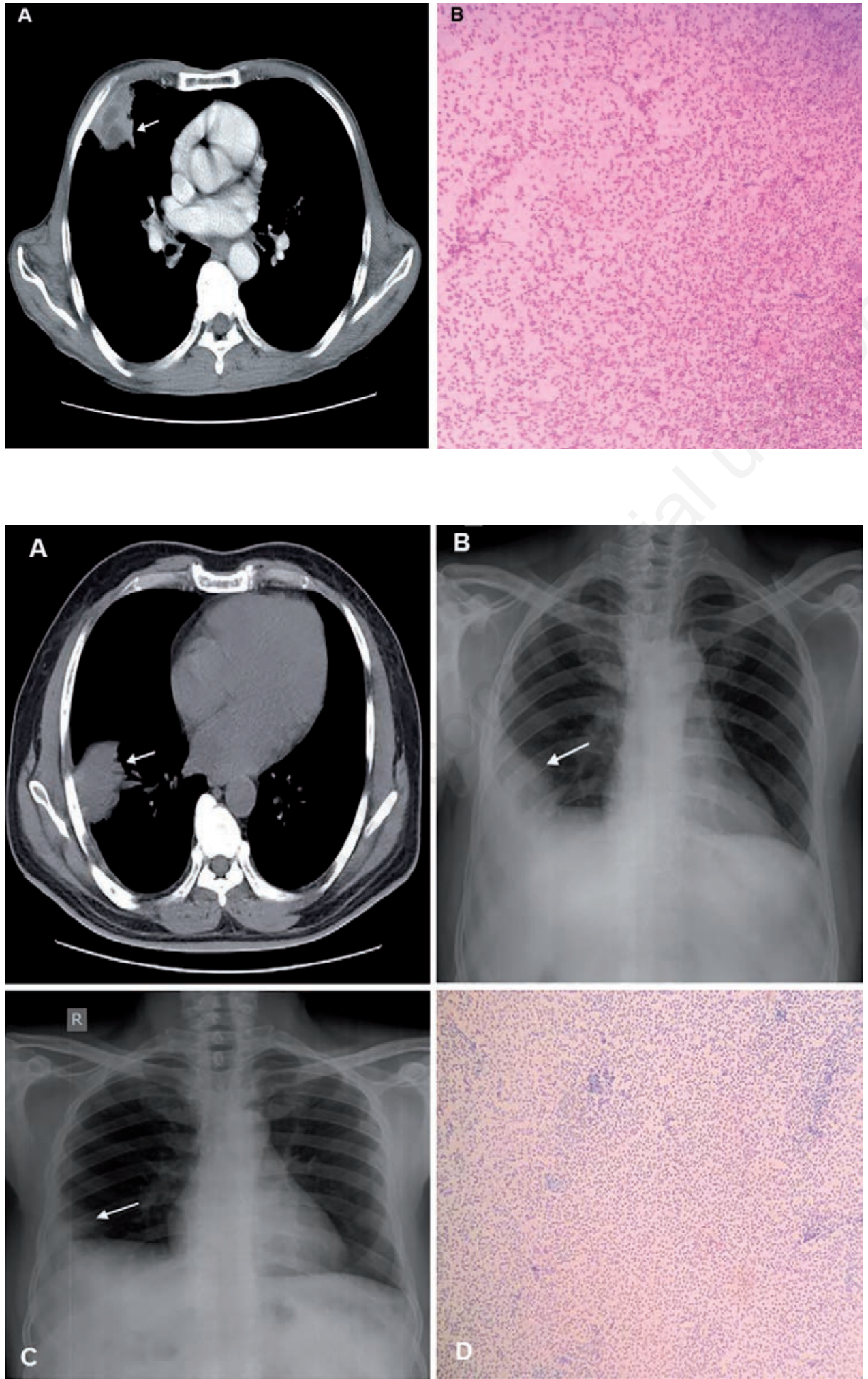

Figure 5. Imaging and histopathological features of NMLP-2. A) Axial CT image showing mass lesion with irregular borders with central necrosis in right upper lobe of the lung (white arrow). 5) Smear cytology image of the aspirate from the lesion showing sheets of neutrophils suggestive of lung abscess.

Figure 6. Imaging and histopathological features of NMLP-3. A) Axial CT image showing mass lesion in right lower lobe of the lung (white arrow). B) Chest $\mathrm{X}$ ray showing mass lesion in right lower lobe of the lung (white arrow). C) Chest $X$ ray showing near complete resolution of the lesion following anti-microbial therapy (white arrow). D) Smear cytology of the aspirate from the lesion showing sheets of neutrophils. 
indicative of pulmonary tuberculosis in the past. These studies suggested the scar and the related chronic inflammation as possible mechanisms for cancer development at later stages, most likely due to activating EGFR mutations [15,16]. However, in contrast to these reports, none of the three subjects in our study had past history of TB and / or evidence of scar in the lungs. Nucleic acids are released into blood stream, usually during the process of cell apoptosis or necrosis. This process is not specific to malignant cells, even normal cells release such nucleic acids. However, the load of these circulating nucleic acids is usually higher in subjects with cancer. Although this phenomenon has been known for several decades, the technological advancements in recent years have now made it possible to isolate these circulating nucleic acids. An ultra-sensitive technique like ddPCR is very useful in detecting such cell free nucleic acids irrespective of the pathology in question.

To the best of our knowledge, this is the first report on detection of EGFR hotspot mutations in cfDNA in subjects having nonmalignant lung pathologies. We couldn't find any data on the presence of these mutations in general population irrespective of the smoking status. However, the presence of lymphoma-associated $t(14 ; 18)$ translocation in general population has been reported [21]. Similarly, another recent study revealed the presence of mutations in EGFR exon 19 and exon 20 in bronchiolar adenoma, which are putatively benign clonal proliferations with overlapping morphological features of ciliated muconodular papillary tumors [22]. The presence of EGFR mutations in such non-malignant conditions strongly suggests advanced research in this field to understand the possible implications of such mutations. Several mutations in $E G F R$ are known to be oncogenic in nature. In this study, the patients who showed the presence of EGFR mutations were smokers and had non- malignant inflammatory conditions. Since chronic inflammation is known to induce genetic alterations, we propose that inflammation may be the trigger for the occurrence of these mutations in the background of strong risk factors such as cigarette smoking. We are also of the opinion that such cases should be subjected to long-term follow up as the possibility of them developing malignant tumours in due course of time cannot be ruled out. Although we encountered only three cases, it would be interesting to screen more cases of such non-malignant pathologies for these target mutations and determine conclusively if these mutations are functional liaisons or coincidental associations.

\section{References}

1. Pao W, Girard N. New driver mutations in non-small-cell lung cancer. Lancet Oncol 2011;12:175-80.

2. Ma J, Li N, Guarnera M, Jiang F. Quantification of plasma miRNAs by digital PCR for cancer diagnosis. Biomark Insights 2013;8:127-36.

3. Shigematsu H, Lin L, Takahashi T, et al. Clinical and biological features associated with epidermal growth factor receptor gene mutations in lung cancers. J Natnl Cancer Inst 2005;97:339-46.

4. Forshew T, Murtaza M, Parkinson C, et al. Non-invasive identification and monitoring of cancer mutations by targeted deep sequencing of plasma DNA. Sci Transl Med 2012;4:136ra68.

5. Oxnard G, Paweletz C, Kuang Y, et al. Noninvasive detection of response and resistance in EGFR mutant lung cancer using quantitative next-generation genotyping of cell-free plasma DNA. Clin Cancer Res 2014;20:1698-705.

6. Kobayashi S, Boggon TJ, Dayaram T, et al. EGFR mutation and resistance of non-small cell lung cancer to gefitinib. N Engl J Med 2005;352:786-92.

7. Sharma SV, Bell DW, Settleman J, Haber DA. Epidermal growth factor receptor mutations in lung cancer. Nat Rev Cancer 2007; 7:169-81.

8. Li K, Yang M, Liang N, Li S. Determining EGFR-TKI sensitivity of G719X and other uncommon EGFR mutations in nonsmall cell lung cancer: Perplexity and solution (Review). Oncol Rep 2017;37:1347-58.

9. Crowley E, Nicolantonio F, Loupakis F, Bardelli A. Liquid biopsy: monitoring cancer- genetics in the blood. Nat Rev Clin Oncol 2013;10:472-84.

10. Santarpia M, Liguori A, D’Aveni A, et al. Liquid biopsy for lung cancer early detection. J Thorac Dis 2018;10:S882-97.

11. Zhang R, Chen B, Tong X, et al. Diagnostic accuracy of droplet digital PCR for detection of EGFR T790M mutation in circulating tumor DNA. Cancer Manag Res 2018;10:1209- 18.

12. Watanabe M, Kawaguchi T, Isa S, et al. Ultra-sensitive detection of the pre-treatment EGFR T790M mutation in non-small cell lung cancer patients with an EGFR-activating mutation using droplet digital PCR. Clin Cancer Res 2015;21:3552-60.

13. Zhang B, Xu C, Shao Y, et al. Comparison of droplet digital PCR and conventional quantitative PCR for measuring EGFR gene mutation. Exp Ther Med 2015;9:1383-8.

14. Hindson B, Ness K, Masquelier D, et al. High-throughput droplet digital PCR system for absolute quantitation of DNA copy number. Anal Chem 2011;83:8604-10.

15. Hwang IK, Paik SS, Lee SH. Impact of pulmonary tuberculosis on the EGFR mutational status and clinical outcome in patients with lung adenocarcinoma. Cancer Res Treat 2019;51:158-68.

16. Luo $\mathrm{YH}, \mathrm{Wu} \mathrm{CH}, \mathrm{Wu}$ WS, et al. Association between tumor epidermal growth factor receptor mutation and pulmonary tuberculosis in patients with adenocarcinoma of the lungs. J Thorac Oncol 2012;7:299-05.

17. Bell DW, Gore I, Okimoto RA, et al. Inherited susceptibility to lung cancer may be associated with the T790M drug resistance mutation in EGFR. Nat Genet 2005;37:1315- 6.

18. Girard N, Lou E, Azzoli CG, et al. Analysis of genetic variants in never-smokers with lung cancer facilitated by an internetbased blood collection protocol: A preliminary report. Clin Cancer Res 2010;16:755-63.

19. Yu HA, Arcila ME, Harlan M, et al. Germline EGFR T790M mutation found in multiple members of a familial cohort. J Thorac Oncol 2014;9:554-8.

20. Lou Y, Pecot CV, Tran HT, et al. Germline mutation of T790M and dual/multiple EGFR mutations in patients with lung adenocarcinoma. Clin Lung Cancer 2016;17:e5-11.

21. Nambiar M., Raghavan S.C. Prevalence and analysis of $\mathrm{t}(14 ; 18)$ and $\mathrm{t}(11 ; 14)$ chromosomal translocations in healthy Indian population. Ann Hematol 2010;89:35-43.

22. Chang JC, Montecalvo J, Borsu L, et al. Bronchiolar adenoma: Expansion of the concept of ciliated muconodular papillary tumors with proposal for revised terminology based on morphologic, immunophenotypic, and genomic analysis of 25 cases. Am J Surg Pathol 2018;42:1010-26. 